\title{
Frontières
}

\section{La mort en chiffres}

\section{Marc Termote}

Volume 18, numéro 2, printemps 2006

La mort dans tous ses états

URI : https://id.erudit.org/iderudit/1073219ar

DOI : https://doi.org/10.7202/1073219ar

Aller au sommaire du numéro

Éditeur(s)

Université du Québec à Montréal

ISSN

1180-3479 (imprimé)

1916-0976 (numérique)

Découvrir la revue

\section{Citer cet article}

Termote, M. (2006). La mort en chiffres. Frontières, 18(2), 37-40. https://doi.org/10.7202/1073219ar

\section{Résumé de l'article}

Cet article tente de répondre brièvement à des questions comme : pourquoi recueillir des données sur les décès ? que faut-il entendre par espérance de vie ? où le Québec se situe-t-il en matière de mortalité ? qu'en est-il de l'inégalité devant la mort, entre autres de la différence entre hommes et femmes ? quelles sont les principales causes de décès ? quel impact ces causes ont-elles sur l'espérance de vie ? quelles sont les perspectives d'avenir?
Ce document est protégé par la loi sur le droit d'auteur. L’utilisation des services d’Érudit (y compris la reproduction) est assujettie à sa politique d'utilisation que vous pouvez consulter en ligne.

https://apropos.erudit.org/fr/usagers/politique-dutilisation/ 


\section{LA MORT EN CHIFFRES}

\section{Résumé}

Cet article tente de répondre brièvement à des questions comme: pourquoi recueillir des données sur les décès? que faut-il entendre par espérance de vie? où le Québec se situe-t-il en matière de mortalité? qu'en est-il de l'inégalité devant la mort, entre autres de la différence entre hommes et femmes? quelles sont les principales causes de décès? quel impact ces causes ont-elles sur l'espérance de vie? quelles sont les perspectives $d$ 'avenir?

Mots clés: statistiques de décès espérance de vie - inégalité devant la mort-causes de décès.

\section{Abstract}

In this paper, we try to briefly answer questions such as: why collect mortality data? what is the meaning of a "life expectancy"? how does Quebec's mortality regime compare with the one observed in other countries? what about inequality with respect to death, for instance disparities between males and females? what are the main causes of death? how do these causes affect life expectancy? what may we expect for the future?

Keywords: mortality data - life expectancy - inequality with respect to death - causes of death.
Marc Termote, Ph.D., professeur titulaire,

Institut national de la recherche scientifique (INRS-UCS).

\section{POURQUOI COMPTER LES MORTS?}

$\mathrm{Au}$ cours des millénaires, les êtres humains ont pris l'habitude de se compter. Dans les grandes civilisations antiques, les gouvernants avaient déjà compris l'importance de procéder plus ou moins régulièrement à un recensement de leur population. Qu'il s'agisse des Égyptiens, des Babyloniens ou des Chinois, connaître le nombre et les caractéristiques des personnes vivant sur le territoire était considéré comme un acte fondamental de souveraineté. La puissance de l'État, donc du souverain, était liée au nombre d'habitants qui lui étaient soumis, et donc au nombre de guerriers dont le souverain disposait. Chez les Chinois, par exemple, cette obsession du grand nombre était telle que Confucius a dû rappeler, dans ses Pensées, que «Nos souverains devraient se préoccuper moins du nombre de leurs sujets que de leur richesse».

Sans doute est-ce parmi les Hébreux que cette dimension à la fois religieuse et mili- taire des recensements des vivants était la plus articulée. Le quatrième livre du Pentateuque (ce dernier terme désigne les cinq livres sacrés attribués, selon la tradition, à Moïse) est significativement intitulé «Livre des Nombres » par les traducteurs grecs de la Torah. Selon ce Livre, au moins deux recensements ont été effectués sur l'ordre direct de Dieu, afin d'énumérer « d'après la lignée paternelle, toute la communauté des Israélites, selon leur famille, tête par tête, à partir de l'âge de 20 ans, c'est-à-dire tous les hommes qui pourront aller à la guerre en Israël» (Nombres, 1-4 et 26). La référence au lignage paternel illustre bien la signification religieuse du recensement: il s'agit de remonter à la source même du peuple élu par Dieu, selon la descendance d'Abraham à Isaac, à Jacob, aux fils de Jacob et de là aux tribus d'Israël. On remarquera que les femmes n'étaient pas jugées dignes d'être recensées: elles ne pouvaient ni accéder à la propriété de la terre, ni «aller à la guerre».

Depuis la plus haute antiquité, on comptait donc le nombre de vivants. Mais on ne se préoccupait guère du nombre de morts. Seul le décès des souverains et des digni-

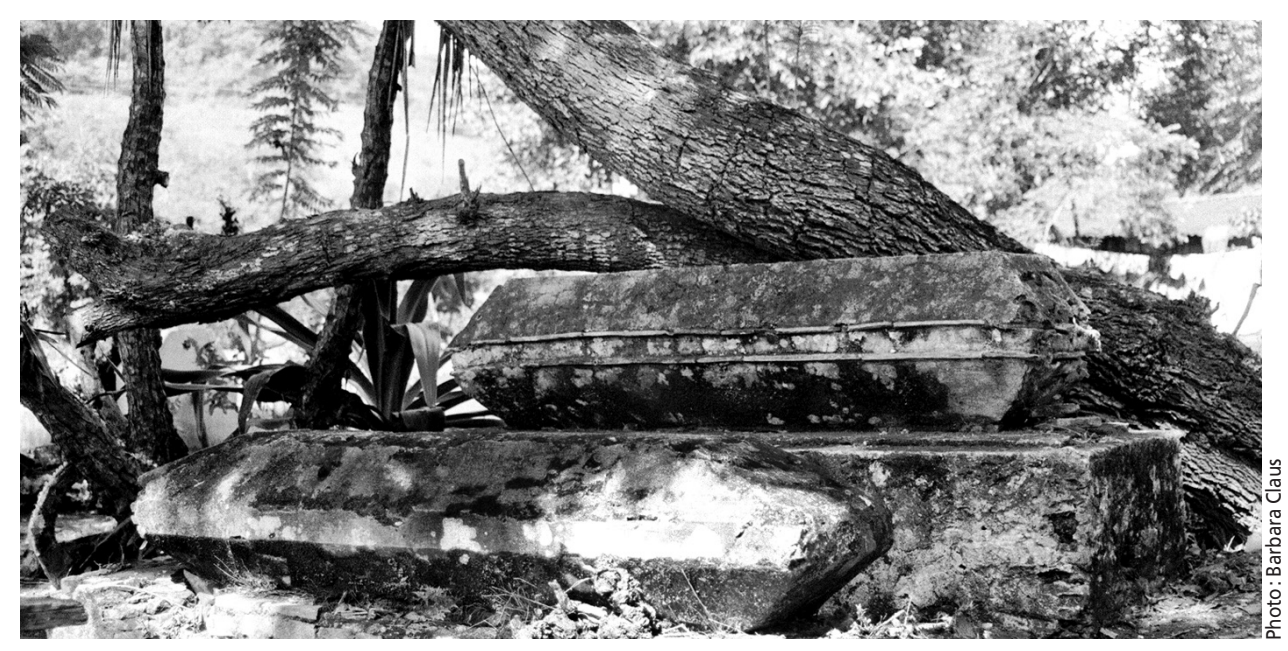


taires était digne d'intérêt. La mortalité (tout comme la fécondité d'ailleurs) était considérée comme un phénomène essentiellement exogène, sur lequel on n'avait guère de prise. Dans certaines sociétés antiques, la mort (comme la naissance) était cependant aussi l'occasion de rendre tribut aux divinités, selon une tarification parfois très détaillée. C'est ainsi que chez les Romains on estimait le nombre et les caractéristiques des décédés, en se basant sur le montant des offrandes faites aux dieux lors d'un décès. Cette manière indirecte de comptabiliser les décès ne permettait évidemment pas de saisir la totalité des décès, loin de là.

La mortalité était considérée comme un phénomène exogène, un «acte de Dieu», mais elle exerçait aussi une fonction de régulation des populations. Depuis les origines jusqu'au XVIII ${ }^{\mathrm{e}}$ siècle, les sociétés humaines ont toutes connu à la fois une forte fécondité et une forte mortalité, car elles ne disposaient d'aucun moyen efficace pour maîtriser la première ou lutter contre la seconde. Il en résultait un accroissement naturel (différence entre le nombre de naissances et le nombre de décès) sans doute extrêmement faible, mais qui, s'il s'étendait sur une longue période, finissait par exercer un impact négatif sur le rapport populationsubsistance. Pour rétablir l'équilibre, il fallait «nécessairement» passer par des crises de surmortalité: les épidémies, les famines, les guerres, jouaient ce rôle de régulation. Ces trois régulateurs « exogènes» sont précisément ceux que Dieu a proposés au roi David comme pénitence pour avoir commis le péché d'orgueil en ordonnant un recensement pour «mesurer sa propre gloire et puissance», et non celles de Dieu. Selon le chapitre 24 du Deuxième livre de Samuel, David avait le choix entre sept ans de famine, trois mois de pertes devant l'ennemi, et trois jours de peste: il choisira cette dernière formule.

Tant que la mortalité était considérée comme un phénomène exogène, résultant de la volonté divine, tant que les êtres humains n'étaient pas capables de lutter contre la mortalité, tant que le nombre de décès était plus ou moins égal au nombre de naissances, compter les morts n'était guère utile: on savait que, sur une période relativement brève, soit environ un quart de siècle (jusqu'au milieu du XVIII ${ }^{\mathrm{e}}$ siècle, la durée moyenne de la vie dans la plupart des pays européens ne dépassait guère 25 ans), il suffisait d'enregistrer le nombre de naissances pour connaître plus ou moins le nombre de décès. Mais une fois que l'on a commencé à comprendre que l'on pouvait agir sur la mortalité, une fois que l'on a commencé à comprendre que l'on pouvait contenir les épidémies, voire les enrayer et les prévenir, une fois que l'on a commencé à connaître les processus de contagion et les principes de l'hygiène, une fois que la médecine a commencé à se développer en traitant les causes plutôt que les effets et en mettant au point une médication plus adéquate, alors il devenait important de connaître non seulement le nombre des vivants, mais aussi celui des morts, plus précisément, de connaître les morts pour permettre aux vivants de vivre.

Connaître les morts, cela ne signifie pas seulement compter le nombre de décès, mais cela implique qu'il faut aussi comptabiliser ces décès selon le sexe de la personne décé-

\section{À TOUS LES ÂGES, \\ LA PROBABILITÉ DE DÉCÉDER \\ DANS L'ANNÉE \\ EST NETTEMENT SUPÉRIEURE \\ À CELLE DE GAGNER AU BINGO}

\section{OU À LA LOTERIE.}

dée, son âge, son statut social, son statut professionnel, son revenu, éventuellement sa religion, sa langue, son groupe ethnique et son appartenance culturelle, et bien sûr, selon la cause (immédiate et indirecte) du décès. Pour pouvoir allonger la durée de vie des vivants, pour être capable de lutter contre la mortalité, il faut connaître les conditions dans lesquelles les vivants ont vécu et celles dans lesquelles ils meurent.

Il aura fallu attendre la seconde moitié du XVII ${ }^{\mathrm{e}}$ siècle pour que, au-delà du simple comptage du nombre de décès, on cherche à dégager les conditions dans lesquelles les gens décédaient, pour pouvoir éventuellement agir sur celles-ci. En 1662, John Graunt compare le régime de mortalité observé à Londres avec celui que connaissait une paroisse rurale anglaise, et en 1692 Edmund Halley (l'astronome qui a donné son nom à une comète) établit la première table de mortalité, en mettant en relation le nombre de décès dans la ville polonaise de Wroclaw et l'âge des décédés, pour en dériver des probabilités de décès selon l'âge, ce qui permettra ultérieurement d'estimer l'espérance de vie, c'est-à-dire le nombre d'années que l'on peut espérer vivre si les probabilités de décès ne changent pas. Ces deux scientifiques sont considérés comme les fondateurs de ce que l'on appellera plus tard (le terme apparaît pour la première fois en 1855) la démographie, c'est-à-dire la science de la population. Voyons maintenant ce que cette "comptabilité des décès » nous enseigne sur les conditions de mortalité au Québec.

\section{LA COMPTABILITÉ MORTUAIRE AU QUÉBEC}

$\mathrm{Au}$ Québec, comme dans la plupart des pays, tout décès doit obligatoirement être déclaré à l'état civil: aucun cadavre ne peut être inhumé ou incinéré sans un certificat de décès dûment rempli et signé par le médecin qui a constaté le décès. Ce certificat est un document public qui, selon la législation canadienne, doit obligatoirement contenir les renseignements suivants: le nom de la personne décédée, sa date de naissance, son sexe et son lieu de résidence, la cause du décès, le lieu du décès, la date et l'heure approximative du décès; en outre, au Québec, il y a lieu de mentionner le lieu de naissance ainsi que la langue maternelle et la langue d'usage de la personne décédée.

En ce qui concerne la cause du décès, le certificat de décès doit distinguer la cause «immédiate» du décès, les causes «initiales» ( «antécédents») du décès et les «autres états morbides importants» ayant contribué au décès. Par exemple, sur le certificat de décès d'une personne souffrant d'une bronchite chronique qui décède d'urémie causée par une maladie rénale chronique, on indiquera l'urémie comme cause de décès immédiate, la maladie rénale chronique comme cause initiale et la bronchite chronique comme autre état morbide important.

Les informations contenues dans ce certificat sont inscrites dans le registre de l'état civil. Le dépouillement périodique de ce registre permet de connaître le nombre de décès, les causes de décès et certaines caractéristiques des personnes décédées.

Le nombre de décès augmente régulièrement: en 1900 il y avait 33000 décès au Québec, et ce chiffre est passé à 43000 en 1981, puis à 55000 en 2003. Une telle croissance n'est guère surprenante, d'abord parce que le nombre d'habitants a augmenté, et ensuite parce que la population a considérablement vieilli. Pour tenir compte de l'augmentation de la population, on peut rapporter le nombre de décès au nombre d'habitants. Ce rapport, appelé taux de mortalité, a d'abord fortement baissé, de 21 décès pour mille habitants en 1900 à $6,5 \%$ en 1981, mais au cours des 20 dernières années il a lentement augmenté (il est actuellement proche de 7,5\%0). Ce renversement de tendance signifie que désormais les gains (en termes de nombre de décès) réalisés grâce à la lutte contre la mortalité sont dominés par les effets négatifs dus à l'évolution démographique, particulièrement ceux qui résultent du vieillissement de la population.

Pour pleinement capter l'impact des facteurs démographiques sur la mortalité, il ne suffit cependant pas de rapporter le nombre de décès au nombre d'habitants. 
La mortalité est en effet un processus très peu démocratique: elle ne frappe pas avec la même intensité les hommes et les femmes, et elle discrimine fortement selon l'âge. Dans ces conditions, il nous faut rapporter le nombre de décès observés à un âge donné pour un sexe particulier, au nombre de personnes de cet âge et de ce sexe. On constate alors qu'à tous les âges, le risque de décéder dans l'année est supérieur chez les hommes. Typiquement, la relation entre risque de décès et âge se présente selon une courbe en U. La mortalité infantile demeure assez élevée au Québec: actuellement, d'une année à l'autre, entre 4 et 5 nouveau-nés sur 1000 décéderont dans l'année qui suit leur naissance (un peu plus chez les garçons, un peu moins chez les filles), mais une fois franchie cette première année, le risque de décès devient très faible (à peine 1 ou 2 décès sur 10000) et ce jusqu'à l'âge de 15 ans. À partir de cet âge, la mortalité remonte légèrement chez les femmes (jusqu'à 6 décès pour 10000 chez celles de 35-39 ans), mais plus rapidement chez les hommes (13 décès pour 10000). Ensuite, l'augmentation du risque de décès est régulière et de plus en plus rapide: entre 60 et 64 ans, un homme a une «chance» sur 100 de décéder dans l'année, une femme presque deux fois moins; entre 70 et 74 ans, le risque est de 3 pour cent chez les hommes, la moitié chez les femmes; entre 80 et 84 ans, il est de 8 pour cent chez les hommes et de 5 pour cent chez les femmes; etc. À tous les âges, la probabilité de décéder dans l'année est nettement supérieure à celle de gagner au bingo ou à la loterie. À partir d'un certain âge, nous aurions sans doute intérêt, lorsque nous nous levons le matin, à nous dire qu'il existe un risque non négligeable que nous ne soyons plus de ce monde l'an prochain. Qui d'entre nous prendrait un avion qui a une chance sur 100 de s'écraser? Et cependant, c'est à ce niveau de risque que vit un Québécois une fois qu'il a atteint la soixantaine...

La mortalité infantile est souvent considérée comme un indicateur important du niveau de développement d'un pays. À cet égard, le Québec ne fait pas mauvaise figure: avec 4,6 décès dans l'année qui suit la naissance pour 1000 naissances, le Québec fait nettement mieux que le reste du Canada (6 décès pour 1000 naissances) et les États-Unis (7 décès pour 1000 naissances). Mais des progrès restent à faire: en France, en Allemagne, au Danemark, en Finlande, en Norvège, en République tchèque, en Slovénie, on observe 4 décès pour 1000 naissances, au Japon, en Suède, à Singapour, à Hong Kong, 3 décès pour 1000 naissances, et en Islande, 2 décès pour 1000 naissances. À l'autre extrême, dans de nombreux pays du tiers-monde, la mortalité infantile dépasse encore nettement 100 décès pour 1000 naissances (en Angola,
20\% des nouveau-nés n'atteignent pas leur premier anniversaire).

Une manière de résumer en un seul chiffre les conditions de mortalité observées à chaque âge est de supposer qu'un enfant qui vient de naître sera soumis à chaque âge de sa vie au risque de décès observé actuellement à chacun de ces âges, et de calculer ainsi le nombre d'années que ce nouveau-né peut espérer vivre si les conditions actuelles de mortalité ne changent pas. Ce nombre attendu d'années à vivre est ce que l'on appelle l'espérance de vie à la naissance (cette mesure, qui peut aussi se calculer, non à partir de la naissance, mais à partir de tout âge subséquent, a ensuite été étendue en termes d'espérance de vie en bonne santé, en état d'autonomie, etc.). Selon les derniers chiffres disponibles (ceux de 2001-2003), l'espérance de vie à la naissance s'élève au Québec à 76,8 ans pour les hommes et 82,1 ans pour les femmes. Dans le reste du Canada, l'espérance de vie est légèrement supérieure chez les hommes (par exemple, elle est de 77,7 années en Ontario), mais elle est pratiquement identique chez les femmes.

Le régime de mortalité que connaissent les hommes québécois situe le Québec dans le peloton de tête: avec une espérance de vie de 78 ans, seuls l'Islande et le Japon nous dépassent nettement. Pour les hommes, les conditions québécoises sont nettement plus favorables que celles que connaissent plusieurs autres pays développés: l'espérance de vie n'est que de 75 années aux ÉtatsUnis et dans la plupart des pays d'Europe occidentale et centrale, et dans la plupart des pays d'Europe orientale elle se situe aux alentours de 65 ans (en Russie, elle n'est que de 59 ans). Dans certains pays africains, particulièrement ceux où sévit le sida, l'espérance de vie des hommes n'atteint même pas 40 ans. L'image est un peu différente lorsqu'il s'agit de l'espérance de vie des femmes: trois pays nous dépassent à cet égard, soit le Japon (84 ans), la Suisse et la France (83 ans).

Dans presque tous les pays, l'espérance de vie des femmes est supérieure à celle des hommes, l'écart étant en moyenne de 4 à 5 ans. Les seules exceptions sont le Bangladesh, le Burkina Faso, le Niger, la Syrie (où il y a égalité), et l'Afghanistan, la Namibie, le Népal et le Zimbabwe (où les femmes ont une espérance de vie inférieure à celle des hommes). L'écart entre hommes et femmes est particulièrement élevé dans certains pays d'Europe orientale: en Biélorussie, en Ukraine, en Russie, l'avantage des femmes atteint environ 12 ans.

Les différences internationales en matière d'espérance de vie (tous sexes confondus) peuvent donc être considérables (du simple au double). Mais si les disparités à l'intérieur d'un pays sont en général nettement plus faibles, elles peuvent dans certains cas être significatives. Ainsi, lorsqu'il s'agit du Canada, l'écart entre provinces est aujourd'hui relativement faible (environ une année), mais à l'intérieur de la région métropolitaine de Montréal les différences sont considérables: l'espérance de vie dans le «croissant d'or » (Westmount, Outremont, Mont Royal, etc.) dépasse de huit années celle du Centre-Sud. Une telle inégalité démontre bien à quel point les conditions socioéconomiques peuvent influencer la mortalité.

Les progrès enregistrés au cours des 150 dernières années en matière de lutte contre la mortalité ont été impressionnants. Durant l'Antiquité, le Moyen-Âge et jusqu'au milieu du XVIII ${ }^{\mathrm{e}}$ siècle, dans la plupart des pays européens, l'espérance de vie ne dépassait guère 25 ans. Grâce aux progrès de la médecine et de l'hygiène, et avec l'amélioration des conditions de vie, elle a ensuite augmenté significativement pour atteindre environ 35 ans au milieu $\mathrm{du} \mathrm{XIX}^{\mathrm{e}}$ siècle, soit un gain moyen d'une année d'espérance de vie par décennie, ce qui est appréciable. Un siècle plus tard, en 1950, l'espérance de vie au Québec et dans la plupart des pays développés se situait aux alentours de 67 ans, soit un gain moyen de trois années par décennie. La progression s'est donc fortement accélérée pendant cette période. Au cours du dernier demi-siècle, les gains réalisés en matière d'espérance de vie sont moindres, mais restent considérables: en moyenne l'espérance de vie augmente d'un an tous les cinq ans. À ce rythme, l'espérance de vie atteindra 90 ans dans 50 ans et à la fin de ce siècle, un nouveau-né pourra espérer devenir centenaire.

Il importe de souligner qu'aujourd'hui, lorsqu'on dit que l'espérance de vie d'un nouveau-né atteint 80 ans (tous sexes confondus), on sous-estime en réalité l'âge qu'aura ce nouveau-né lorsqu'il décédera. Cette espérance de vie est en effet une mesure du régime actuel de mortalité, et elle ne tient pas compte des progrès qui seront réalisés en matière de lutte contre la mortalité pendant toute la durée de la vie de ce nouveau-né.

Lorsqu'on commence à envisager une espérance de vie de 100 ans pour nos arrière-petits-enfants, on est tout naturellement amené à se poser la question de la durée maximale de la vie humaine, ce que l'on appelle la longévité. Lorsqu'on se base sur des âges dûment vérifiés, il semble bien que nulle part la vie humaine n'a dépassé 125 ans. Si l'on en croit certains travaux sur le vieillissement cellulaire et sur l'horloge génétique, on ne peut cependant exclure que cette limite imposée à l'Homo sapiens par la biologie ne soit un jour dépassée. 
Tout ce qui vit doit mourir. L'espèce humaine, au même titre que les autres espèces vivantes, est soumise à cette nécessité biologique. Comme nous l'avons $\mathrm{vu}$, cette nécessité se manifeste cependant selon des modalités diverses: la mortalité varie selon le sexe, l'âge, les régions et les pays, les époques, etc. Puisque ces variations ne sont pas dues au hasard, peut-on tenter de dégager ce qui dans la mort est nécessité et ce qui est dû aux conditions de vie, au comportement humain? L'analyse des causes de décès permet d'apporter quelques éléments de réponse à cette question.

Le fait qu'il y ait apparemment une limite extrême à la durée de la vie humaine, joint à l'observation selon laquelle à partir d'un certain âge les risques de décès augmentent exponentiellement, ouvre une piste de réflexion. On peut en effet avancer que la mortalité est composée de deux phénomènes, l'un correspondant à un processus d'usure biologique, et l'autre étant fonction de circonstances extérieures, ayant pour effet de devancer «anormalement» l'âge de la mort. On distinguera donc deux grands groupes de causes de décès: d'une part, les causes qui sont du domaine de la «nécessité» et qui sont donc inéluctables (essentiellement les déficiences congénitales et les processus de dégénérescence, tels que les cancers, les maladies cardio- et cérébrovasculaires, certaines maladies chroniques) et, d'autre part, celles qui au contraire pourraient être évitées par des mesures sanitaires et sociales (maladies infectieuses, malnutrition, toxicomanie, accidents, etc.). Les premières seront qualifiées d'endogènes, les secondes d'exogènes. Une telle dichotomie doit cependant être nuancée: les causes endogènes peuvent également faire l'objet d'interventions visant, sinon à les empêcher, du moins à en retarder l'apparition ou à en ralentir l'évolution.

Depuis 2000, les causes de décès sont codées selon une nouvelle version, la dixième, de la classification internationale des maladies, qui comprend 8000 catégories (au lieu de 5000 précédemment), ce qui rend délicate l'analyse de l'évolution de longue période. Par ailleurs, on constate des fluctuations importantes dans l'évolution au cours de courte période, du moins pour certaines causes de décès (c'est le cas, par exemple, pour les "décès par accident de véhicule» et pour la grippe). Il faut donc être prudent lorsqu'on tente de dégager des tendances.
À l'aube de ce siècle, au Québec comme dans les autres sociétés dites développées, la majorité (environ les deux tiers) des décès est due à des causes endogènes, particulièrement les tumeurs, qui sont la cause d'environ un tiers des décès, et les maladies de l'appareil circulatoire (cardiopathies, maladies cérébrovasculaires), qui sont responsables de quelque $30 \%$ des décès. Les principales causes de décès sont relativement similaires chez les hommes et les femmes. Les maladies parasitaires et infectieuses (dont le sida) ne représentent plus qu'une part infime des causes de décès: environ un pour cent. Avec l'effondrement de la mortalité infectieuse, la mortalité cardiovasculaire a d'abord pris le relais, mais depuis les années 1950 celle-ci a connu à son tour un déclin marqué. Cette dernière évolution illustre bien à quel point les progrès dans la lutte contre la mort se manifestent également, et de plus en plus, dans le domaine des causes dites endogènes, liées au vieillissement et à la dégénérescence.

Certaines causes de décès méritent une attention particulière, même si elles ne comptent que pour une faible partie du total des décès. Sur les quelque 55000 décès enregistrés ces dernières années au Québec, environ 700 sont dus à des accidents de véhicules à moteur et 1300 à des suicides, ces derniers étant 10 fois plus nombreux que les homicides ou les décès dus au VIH (sida). Même si ces quatre causes de décès représentent donc à peine 4\% du total, elles sont dignes d'intérêt, non seulement parce qu'il s'agit de décès "évitables», mais également parce qu'elles frappent surtout de jeunes adultes. Elles ont donc un impact considérable sur l'espérance de vie. Tout comme la lutte contre la mortalité infantile a permis jusqu'à récemment (car il semble bien qu'à cet égard on soit proche de la limite minimale) des gains importants en matière d'espérance de vie, de même la lutte contre la mortalité «exogène» parmi les jeunes adultes ne peut que contribuer significativement à l'augmentation de la durée moyenne de vie. "Guérir» d'un cancer une personne âgée de 80 ans a peu d'impact sur l'espérance de vie, contrairement à "prévenir» un projet de suicide d'un jeune de 20 ans

La mise en relation de l'âge au décès et des causes de décès a conduit au concept d' "années potentielles de vie perdues». On a ainsi pu estimer que si, demain, toutes les formes de cancer étaient "guéries», cela ne permettrait de gagner au mieux qu'environ 3 à 4 années d'espérance de vie. Par ailleurs, si demain on parvenait à éliminer toutes les causes exogènes de décès, l'espérance de vie des hommes augmenterait de près de 7 ans et celle des femmes de 4 ans, ce qui impliquerait que l'avantage biologique dont ces dernières bénéficient serait relativement faible. Comme les femmes courent moins de risques de décéder à la suite de causes évitables, elles bénéficient moins des gains que l'on peut réaliser dans ce domaine. Par exemple, si demain tous les Canadiens étaient non-fumeurs, l'espérance de vie des femmes augmenterait de 2 ans, celle des hommes de 4 ans.

On ne peut pas ne pas tenir compte de tels résultats lorsqu'on doit établir des priorités dans les investissements que la société fait en matière de lutte contre la mortalité. "Rien ne vaut la vie», dit-on. Mais la comptabilité des morts nous enseigne que la vie n'a pas la même valeur pour tous, que les inégalités devant la mort sont nombreuses, et que l'on ne peut échapper à la question: «que vaut une vie?». Derrière les chiffres de la mort, il n'y a pas seulement la vie des individus, mais aussi celle de la société. 